\title{
Functional properties of ovotransferrin from chicken egg white and its derived peptides: a review
}

\author{
Ethige Chathura Nishshanka Rathnapala ${ }^{1} \cdot$ Dong Uk $\mathrm{Ahn}^{2} \cdot$ Sandun Abeyrathne $^{1}$ (i)
}

Received: 26 October 2020/Revised: 25 February 2021/ Accepted: 4 March 2021 / Published online: 29 March 2021

(C) The Korean Society of Food Science and Technology 2021

\begin{abstract}
With emerging trends in the food and pharmaceutical industries, potential applications of egg-derived bioactive compounds were recognized. Ovotransferrin is a major egg white functional protein responsible for multiple bioactivities. The objectives of this review are to provide scientific evidence of the functional properties of chicken ovotransferrin and its derived peptides and to identify future research approaches and applications. Various easy, economical, and non-toxic methods have been reported to produce ovotransferrin with high yield and purity, and chemical and enzymatic approaches have been employed to release bioactive peptides. The native ovotransferrin is known to have antimicrobial, antioxidant, anticancer, and immunomodulatory activities. The peptides produced from ovotransferrin also are reported to have antioxidant, antimicrobial, antihypertensive, and anticancer properties. However, little or no application of these compounds in the food and pharmaceutical areas is available yet. Therefore, the practical application of OTF in nutraceutical and pharmaceutical areas are among the emerging areas of research.
\end{abstract}

Keywords Ovotransferrin - Egg white protein - Bioactive peptide $\cdot$ Nutraceutical $\cdot$ Pharmaceutical

Sandun Abeyrathne

sandun@uwu.ac.lk

1 Badulla, Sri Lanka

2 Ames, IA, USA

\section{Introduction}

Eggs play a significant role in the human diet as an affordable nutrient-rich food that sustains human life, health, and growth. With the increase of human health concerns, natural bioactive proteins and peptides received enormous attention from both the consumers as well as the food industries $(\mathrm{Wu}, 2014)$ because scientific evidence proved the existence of bioactive peptides derived from food might have beneficial effects on improving human health and preventing diseases (Möller et al., 2008). Egg contains highly digestible proteins (12\%), lipids (12\%), carbohydrates $(1 \%)$, and traces of vitamins and minerals (1\%) (Kovacs-Nolan et al., 2005). In addition to their use in food processing, egg white proteins such as ovalbumin $(54 \%)$, ovotransferrin $(12 \%)$, ovomucoid $(12 \%)$, lysozyme (3.4\%), and ovomucin (3.5\%) and their derived peptides are highly recognized for their biological activities (Abeyrathne et al., 2013a).

Ovotransferrin from chicken egg white and its derived peptides display a wide range of functional properties. Many methods, including chemical precipitation and chromatography, have been employed to separate ovotransferrin from chicken egg white (Abeyrathne et al., 2014a; 2014b; Ko and Ahn, 2008; Wu and Acero-Lopez, 2012). The native ovotransferrin can carry two irons and exhibits multiple bioactivities, including antimicrobial activity for a wide spectrum of bacteria, fungi, yeasts, and parasites (Ibrahim et al., 1998; Ko et al., 2008a; 2008b; Moon et al., 2012; Valenti et al., 1982), antioxidant activity (Ibrahim et al., 2007; Kim et al., 2012), anticancer activity (Ibrahim and Kiyono, 2009; Moon et al., 2013) and immunomodulatory activity (Xie et al., 2002).

The peptides produced from ovotransferrin using dilute acid hydrolysis (Ibrahim et al., 2000; Kim et al., 2012; 
Moon et al., 2013) or enzymatic hydrolysis (Huang et al., 2010; Keung et al., 1982; Kim et al., 2012; Lee et al., 2006; Lei et al., 2011; Ma et al., 2020; Moon et al., 2017; Shen et al., 2010; Wang et al., 2017) also have been reported to have antioxidant, antimicrobial, antihypertensive and anticancer properties. Hence, egg white ovotransferrin and its derived peptides may have great potentials to be used as antimicrobial and antioxidative agents in the food industry and anticancer, antiviral, antihypertensive, and metal-supplementing agents in the medical and pharmaceutical industries. This review is aimed to investigate the research progress of the functional properties of ovotransferrin from egg white and its derived peptides, and to identify their emerging research and application opportunities in the future.

\section{Structure and chemical properties of ovotransferrin}

Ovotransferrin is a monomeric glycoprotein consists of 686 amino acids with 15 disulfide bonds and no free sulfhydryl groups (Wu and Acero-Lopez, 2012). The molecular mass of ovotransferrin is $78-80 \mathrm{kDa}$ and the isoelectric point is 6.0 (Ibrahim, 2000). Ovotransferrin comprises two globular lobes called N-terminal lobe (residues 1-332) and C-terminal lobe (residues 342-686) that are linked by an alpha helix of 333-341 amino acid residues and interact through noncovalent and hydrophobic interactions (Charter and Largade, 2004). Nine of the 15 disulfide bonds are located in the C-terminal lobe and 6 in the N-terminal lobe (Williams et al., 1982). The two lobes of the ovotransferrin molecule are superposed by a rotation of $167.6^{\circ}$ with a translation of $24.7 \AA$. Each lobe of ovotransferrin is further divided into two subdomains (named as $\mathrm{N} 1, \mathrm{~N} 2$, and $\mathrm{C} 1$, C2) of 160 residues each, and those subdomains are linked through two antiparallel $\beta$-strands (Kurokawa et al., 1995). The N- and C-terminal lobes have homologous amino acid sequences with about $40 \%$ identical residues (Williams et al., 1982). Both of the lobes have the capabilities to reversibly bind one $\mathrm{Fe}^{3+}$ ion with a bicarbonate anion but differ in binding capacities (iron-binding constant of C-terminal lobe: $1.5 \times 10^{8}$; N-terminal lobe: $1.5 \times 10^{14}$ ) (Lin et al., 1994). Also, ovotransferrin can transfer 1 atom of ions such as $\mathrm{Cu}^{2+}$ and $\mathrm{Al}^{3+}$ (Ko and Ahn, 2008). The overall structure of chicken ovotransferrin is identical to those of the human transferrin and rabbit serum transferrin (Kurokawa et al., 1995), and each lobe has an iron-binding site within the interdomain cleft of the lobe (Ibrahim et al., 2000).

The native ovotransferrin is found in two main forms: metal-free (apo) and metal-bound (holo). However, the physicochemical properties of these two forms differ significantly. According to Ko and Ahn (2008), apo-ovotransferrin is colorless and can be easily denatured by physical and chemical treatments, whereas holo-ovotransferrin has a salmon-pink color and shows resistance against thermal denaturation and proteolytic hydrolysis. The isoelectric point of apo-ovotransferrin is 6.73 , whereas that of holo-ovotransferrin is 5.78 (Wenn and Williams, 1968). Furthermore, ovotransferrin has three isoforms; a-ferric (no iron atoms), mono-ferric (carrying one iron atom), and diferric (carrying two iron atoms) (Desert et al., 2001). The ovotransferrin has two putative glycosylation sites in the C-terminal lobe (473-476; Asn-Arg-Thr-Gly and 618-621; Asn-Gly-Ser-Glu) and attached with 4 residues of mannose and 8 residues of $\mathrm{N}$-acetylglucosamine (Williams, 1968). However, Acero-Lopez et al. (2012) reported that highpressure treatment resulted in structural changes of ovotransferrin. At pH 8 and pressure higher than $200 \mathrm{MPa}$, total sulfhydryl groups decreased whereas surface hydrophobicity increased along with partial aggregation. Also, the conformational structure of ovotransferrin was changed from helices, sheets turn, and aggregated strands to intermolecular $\beta$-sheets. However, the structure returned to its original structure at a pressure greater than $500 \mathrm{MPa}$.

\section{Separation and purification of ovotransferrin}

The isolation and purification of egg white proteins have been emerged due to the presence of a broad range of functional properties. Separation techniques such as liquid chromatography that includes gel permeation, ion exchange, and immobilized ligand affinity chromatography were employed most prominently to separate egg white proteins (Chang et al., 2018). Ovotransferrin was first purified using ammonium sulfate at $\mathrm{pH} 3$ (Fraenkel-Conrat and Feeney, 1950). However, the procedure was not easy to scale up because the product purity was low and the amount of ammonium sulfate used was very high. Warner and Weber (1951) fractioned ovotransferrin in crystalline form (both as the iron complex and iron-free protein) using $50 \%$ ethanol in $0.02 \mathrm{M}$ sodium chloride at $\mathrm{pH}$ ranging from 6 to 9 . However, the purity of the product was low. Later, many chromatographic approaches were employed to improve product purity and yield and to minimize the structural alterations during purification ( $\mathrm{Wu}$ and AceroLopez, 2012). Carboxymethyl cellulose (CMC) chromatography was employed to separate ovotransferrin with $0.1 \mathrm{M}$ ammonium acetate (Rhodes et al., 1958) and 0.3 M ammonium sulfate at $\mathrm{pH} 8.5$ (Azari and Baugh, 1967). Further, the use of CMC along with $0.1 \mathrm{M}$ ammonium acetate had advantages such as direct isolation of ovotransferrin without resorting to the preliminary crystallization of other proteins or conventional $\left(\mathrm{NH}_{4}\right)_{2} \mathrm{SO}_{4}$ 
fractionation steps, which enabled the purification of large quantities of ovotransferrin (Azari and Baugh, 1967). Introduction of immobilized metal affinity chromatography (Al-Mashikhi and Nakai, 1987) and DEAE-affinity gel blue chromatography (Chung et al., 1991) significantly improved the purity of the ovotransferrin (94-98\%) as indicated in SDS-PAGE. The development of two-step chromatographic methods such as gel permeation on a Superose 6 Prep Grade column and anion-exchange chromatography on Q Sepharose Fast-Flow column (Awade et al., 1994); frontal chromatography (also called displacement chromatography) on a Q Sepharose Fast Flow column followed by purification by preparative chromatography on the same column) (Croguennec et al., 2001); anion exchange chromatography on Q-Sepharose Fast-Flow column followed by CM-Toyopearl $650 \mathrm{M}$ cation exchange chromatography (Tankrathok et al., 2009), and anion exchange chromatography on Q-Sepharose FastFlow column followed by cation exchange chromatography on SP-Sepharose Fast-Flow column (Omana et al., 2010) further improved the purity of ovotransferrin (75-80\%). Vachier et al. (1995) developed a single anion-exchange chromatography technique using the Q-Sepharose FastFlow column to isolate ovotransferrin with $97.5 \%$ product purity. Non-denaturing two-dimensional electrophoresis (2DE) was employed to separate lysozyme-binding ovotransferrin along with ovalbumin. After separating lysozyme-binding protein, ovotransferrin was extracted using isoelectric focusing (IEF) (Shimazaki and Takahashi, 2018). However, they were good for only laboratory use with no scale-up capability.

A simple, economic protocol and scalable production of apo-ovotransferrin with over $80 \%$ purity and around $99 \%$ yield were proposed by Ko and Ahn (2008). They used $43 \%$ ethanol at $\mathrm{pH} 9.0$ to separate holo-ovotransferrin and ion exchange resin to convert holo-ovotransferrin into the apo-form. Ko and Ahn (2008) further explained that the recovery and stability of ovotransferrin were very high. Therefore, ovotransferrin is more suitable for food applications. The separation methods of multiple proteins from egg white, including ovotransferrin and other egg white proteins such as ovalbumin, ovomucin, and lysozyme using countercurrent chromatography with the polymer-phase system at $\mathrm{pH}$ 8.0, were developed by Shibusawa et al. (1998) and Shibusawa et al. (2001). As a part of the sequential separation method of egg white proteins, Abeyrathne et al. (2013b, 2014a; 2014b) developed a scalable ovotransferrin separation method without using organic solvents (using ammonium sulfate and citric acid), and the method was simpler and more cost-effective than previous methods and had over $85 \%$ purity and over $83 \%$ yield. Recently, Ji et al. (2020) also developed a sequential separation method for 5 major egg white proteins, including ovotransferrin, and reported that the method was easy, rapid, and scalable, and the purity and yield of all 5 proteins were higher than $90 \%$ and $77 \%$, respectively. The development of simple, efficient, and scalable ovotransferrin separation methods is a very important step for the practical application of ovotransferrin and its derived peptides in the nutraceutical and pharmaceutical industries.

\section{Functional properties of ovotransferrin}

\section{Antibacterial activity}

The bacteriostatic activity of ovotransferrin is directly derived from its ability to sequester $\mathrm{Fe}^{3+}$ necessary for microbial growth (Giansanti et al., 2015). The antimicrobial activity of ovotransferrin was first identified by Schade and Caroline (1944). The antimicrobial domain of ovotransferrin was found in the N-lobe (residues 1-332) of ovotransferrin (Ibrahim et al., 1998) and showed antimicrobial activity against a broad spectrum of bacteria, fungi, yeast, and parasites. Ovotransferrin inhibits common foodborne pathogens such as Staphylococcus aureus KCCM 32,395, Bacillus cereus KCCM 40,935, Listeria monocytogenes ATCC 15,313, Escherichia coli 0157:H7 ATCC 43,895, and Helicobacter pylori HpKCTC 26,695 (Moon et al., 2012), and the antibacterial activity of ovotransferrin was dose-dependent. Gram-positive bacteria were more sensitive to ovotransferrin than gram-negative bacteria. Baron et al. (2014) postulated another antibacterial mechanism that is related to the damage of bacterial membrane by ovotransferrin. They reported that ovotransferrin lysed bacterial cell membranes and showed strong antibacterial activity against B. cereus. Bacterial species, including Pseudomonas spp., E. coli, and Staphylococcus mutans, were the most sensitive, whereas Staphylococcus aureus, Proteus spp. and Klebsiella were the most resistant to ovotransferrin (Valenti et al., 1983). Ovotransferrin along with lysozyme and ethylenediaminetetraacetate (EDTA) exhibited strong antibacterial activity against L. monocytogenes (Ko et al., 2008a). The presence of bicarbonate ions increased the bacteriostatic activity of ovotransferrin (Ko et al., 2008b) and the antagonizing effect was reported after adding citrate in the presence of bicarbonate (Valenti et al., 1983). Ibrahim et al. (2000) and Valenti et al. (1987) reported that $\mathrm{Zn}^{2+}$-saturated ovotransferrin exhibited stronger antimicrobial activity than apo-ovotransferrin or other metal-complexes of ovotransferrin. However, Valenti et al. (1987) noted that the direct contact of $\mathrm{Zn}^{2+}$-saturated ovotransferrin with the bacterial surface is required to express desired antibacterial activity. Valenti et al. (1982) reported that ovotransferrin immobilized on Sepharose $4 \mathrm{~B}$ also had the iron-binding 
capacity and showed a bacteriostatic effect on E. coli. Shimazaki and Takahashi (2018) investigated the antibacterial activity of lysozyme-binding ovotransferrin separated with 2DE and IEF and found that it had bacteriolytic activity against $B$. subtilis and E. coli. Cooper et al. (2019) reported that the overexpression of ovotransferrin in chicken embryonic cells improved their antimicrobial activity against Campylobacter jejuni. Also, the ovotransferrin significantly reduced the total aerobic and coliform bacteria in chicken muscle samples stored at $7{ }^{\circ} \mathrm{C}$ for 5 days. Zhu et al. (2019) reported that ovotransferrin enhanced gut health by reducing pathogenic microorganisms such as Helicobacter and Desulfovibrio in guts.

\section{Antioxidant activity}

The ability of ovotransferrin to retard lipid oxidation is derived from its free-radical scavenging activity. Moon et al. (2012) reported that ovotransferrin maintained approximately $60 \%$ of its 2,2-diphenyl-1-picryl hydroxyl (DPPH) radical scavenging capacity for $48 \mathrm{~h}$, but Kim et al. (2012) indicated that approximately $78.2 \%$ of 2,2 diphenyl-2-picrylhydrazyl radical scavenging activity was remaining after $32 \mathrm{~h}$ of incubation. Ovotransferrin exhibited a distinct thiol-linked self-cleavage in a redox-dependent process and the superoxide dismutase-like superoxidescavenging activity of ovotransferrin was promoted by binding copper and manganese over ascorbate or serum albumin (Ibrahim et al., 2007). Hence, the metal-bound (holo) ovotransferrin shows greater dismutation than the metal-free (apo) ovotransferrin. You et al. (2014) reported that when the lysine (residues 327) and glutamic acid (residues 186) in ovotransferrin were conjugates with catechin from green tea, the oxygen radical scavenging capacity of the conjugated ovotransferrin was 4-5 times higher than that of the native one. These results indicated that a slight modification of ovotransferrin is a useful strategy to improve the functional properties of ovotransferrin, but further studies are needed to confirm their effects.

\section{Antifungal and antiviral activity}

Ovotransferrin possessed inhibitory activity against genus Candida. Valenti et al. (1985) tested the antifungal effect of ovotransferrin against over 100 strains of Candida and found that all the Candida strains but $C$. krusei showed sensitivity to ovotransferrin. They also found that iron saturated-ovotransferrin possessed antifungal activity. Although the addition of bicarbonate ions increased the antibacterial activity, it did not inhibit the Candida spp. Valenti et al. (1986) reported that both lactoferrin and ovotransferrin exhibited antifungal activity against $C$.
Albicans through a transferrin adsorption mechanism of $C$. Albicans, but not in C. krusei. The antiviral activity of ovotransferrin against the infection of chicken embryo fibroblast by Marek's disease virus (MDV) and avian herpesvirus was demonstrated by Giansanti et al. (2002) who reported that ovotransferrin was more effective than human transferrin and bovine lactoferrins in inhibiting MDV infection. However, there was no correlation between the antiviral efficacy and the iron saturation of ovotransferrin. Zhou et al. (2018) reported that ovotransferrin suppressed the replication of vesicular stomatitis virus and increased the production of type I interferon in the ovotransferrin-pretreated mouse peritoneal macrophages.

\section{Cytotoxic activity}

Proteins in egg whites play important roles in preventing infections during the development of chicken embryos. Ibrahim and Kiyono (2009) investigated the effect of ovotransferrin on the proliferation of MCF-7 (breast cancer) and HCT-116 (colon cancer) cells and reported that thiol-linked auto-cleaved ovotransferrin exhibited cytotoxic effects against the breast and colon cancer cells. Moon et al. (2013) also tested the cytotoxicity of promod 278P hydrolysate of ovotransferrin against various cancer cell lines, including the larynx (Hep-2), stomach (AGS), lung (SK-MES-1), liver (HepG2), breast (MCF-7), cervix (HeLa) and colon (HT-29) cancer, and found that they had little growth inhibitory effects to those cancer cell lines.

\section{Immunomodulatory activity}

Ovotransferrin is an acute-phase protein, the levels of which increase significantly during inflammation and infections. Xie et al. (2002) reported that ovotransferrin stimulated the production of interleukin-6 (IL-6) and matrix metalloproteinase (MMP) by HD11 cells and augmented phorbol ester-induced respiratory burst activity and degranulation in chickens, and suggested that ovotransferrin modulated macrophage and heterophil functions. Lee et al. (2018) reported that ovotransferrin stimulated the production of nitric oxide in RAW 264.7 macrophages, expression of inducible nitric oxide synthase, and pro-inflammatory cytokines such as tumor necrosis factor- $\alpha$ (TNF- $\alpha$ ), interleukin-1 $\beta$, and Il-6 and increase the phosphorylation level of mitogen-activated protein kinase (MAPK) signaling pathways that are associated with macrophage activation. Thus, ovotransferrin can be used as a natural immunomodulator to enhance immune responses in innate and adaptive immune systems to prevent diseases. Chiurciu et al. (2017) reported that ovotransferrin from hyperimmune chicken eggs possessed immunological 
characteristics similar to immunoglobulin $\mathrm{Y}$ that prevented resistance to antibiotics. Also, ovotransferrin increased major histocompatibility complex class II (MHC-II) and a cluster of differentiation 83 (CD83) levels to enhance maturation of intestinal dendritic cells (DCs). Also, ovotransferrin promoted the expression of cytokines and the genes of TNF- $\alpha$ interferon- $\gamma$ (IFN- $\gamma$ ), interleukin-4 (IL-4), and interleukin-10 (IL-10). Ovotransferrin enhanced the secretion of immunoglobulin $\mathrm{A}(\operatorname{IgA})$ and secretory immunoglobulin A ( $\operatorname{sg} \mathrm{A})$ to promote intestinal humoral immune response and ameliorated the immune dysfunction caused by cyclophosphamide (CP) (Zhu et al., 2018). Zhu et al. (2019) reported that the thymus and spleen index of ovotransferrin- treated mice significantly higher than that of the cyclophosphamide (CP)-treated mice. Also, the secretion of TNF- $\alpha$, IL-10, and IgA in the CP-treated mice returned to normal levels after treating them with ovotransferrin. Shang (2019) reported that egg white ovotransferrin is responsible for enhancing the expression of type I collagen and alkaline phosphate, inhibition of the production of receptor activator of nuclear factor kappa-B (RANKL). (Shang et al, 2020) reported that the supplementation of ovotransferrin regulated the expression of genes that are involved in lipid metabolism, signal transduction, and the endocrine system, and showed that lipoprotein receptor-related protein 1 worked as a scavenger receptor and regulated the ability of ovotransferrin to promote bone formation. Ovotransferrin increased the beneficial microbiota population such as Akkermansia and affected mucosal morphology by increasing crypt depth. The expression of tight junction protein was also increased by supplementation of ovotransferrin in young and adult mice (Zhang et al., 2020).

\section{Production of ovotransferrin-derived functional peptides}

Various approaches to hydrolyze ovotransferrin, including chemical and enzymatic methods, have been reported (Ibrahim et al., 2000; Kim et al., 2012; Lee et al., 2010; Moon et al., 2013). Among the reported methods of hydrolyzing ovotransferrin, enzymatic hydrolysis was more widely used than other methods. Especially, commercially available plant, animal, and fungi or bacterial enzymes have been utilized with the enzyme-to-substrate ratio at 1:25 (Kim et al., 2012) and 1:50 (Moon et al., 2017). Single enzyme hydrolysis was the most commonly used method, but the use of two or more enzyme combinations also have been tested. However, little works were done to produce functional peptides from ovotransferrin using food-grade enzymes such as promod 278P (Table 1).

\section{Functional properties of ovotransferrin-derived peptides}

\section{Antimicrobial activity}

The presence of an antimicrobial domain is a unique characteristic of the transferrin family (Wu and AceroLopez, 2012). A 92 amino acid-residue peptide of ovotransferrin (OTAP-92; Leu109-Asp200) kills E. coli through a membrane damage mechanism and it is capable of destructing Gram-negative bacteria by crossing the outer membrane by a self-promoted uptake that causes damage to the biological function of the cytoplasmic membrane (Ibrahim et al., 2000). Also, bioactive peptides obtained by the hydrolysis of ovotransferrin with trypsin and ficin exhibited antimicrobial effects against $S$. aureus $\left(\mathrm{G}^{+}\right)$and Salmonella typhimurium $\left(\mathrm{G}^{-}\right)$(Zohreh et al., 2014). A novel antimicrobial peptide of ovotransferrin (Ala-GlyLeu-Ala-Pro-Tyr-Lys-Leu-Lys-Pro-Ile-Ala) was reported to have excellent antimicrobial activities against both Gram-positive (S. aureus ATCC 25,923, L. monocytogenes ATCC 19,111, B. subtilis ATC 23,631) and Gram-negative (E. coli ATCC 25,922, P. aeruginosa ATCC 27,553, S. Typhimurium ATCC 50,013) bacteria (Ma et al., 2020). Ovotransferrin contains antiviral peptides that are related to the natural immunity of chickens. Giansanti et al. (2005) reported that hen egg ovotransferrin consisted of two fragments Asp-Gln-Lys-Asp-Glu-Tyr-Glu-Leu-Leu and Lys-Asp-Leu-Leu-Phe-Lys, which contribute to the antiviral activity against Marek's disease virus that infects chicken embryo fibroblasts. Therefore, these protein fragments have a potential application as an antiviral agent.

\section{Antioxidant activity}

Ovotransferrin hydrolysates have been reported to have strong antioxidant activities. Kim et al. (2012) produced ovotransferrin hydrolysates using 10 enzymes and reported that all the hydrolysates had approximately 3.2 to 13.5 times higher superoxide anion scavenging and oxygen radical absorbance capacities than the natural ovotransferrin. Shen et al. (2010) hydrolyzed ovotransferrin with thermolysin and found two tetrapeptides (Trp-Asn-Ile-Pro and Gly-Trp-Asn-Ile) with very high antioxidant activity and postulated that the Trp-Asn-Ile portion could be the core motif responsible for the high antioxidant activity. Also, Huang et al. (2010) obtained a peptide (Ile-Arg-Trp) from ovotransferrin using thermolysin and pepsin and found that this peptide possessed significantly higher oxygen radical absorbance capacity than the whole ovotransferrin. They also suggested that ovotransferrin hydrolysates could be used as a natural antioxidant in 
Table 1 Methods for the hydrolysis of ovotransferrin and its derived activities

\begin{tabular}{|c|c|c|c|}
\hline Method of hydrolysis & Used enzymes or chemicals & Activity & References \\
\hline Enzymatic hydrolysis & Subtilisin & Iron binding & Keung et al. (1982) \\
\hline Acid hydrolysis & $0.03 \mathrm{~N} \mathrm{HCl}$ & Antimicrobial & Ibrahim et al. (2000) \\
\hline Enzymatic hydrolysis & Chymotrypsin & ACE-inhibitory, Antihypertensive & Lee et al. (2006) \\
\hline Enzymatic hydrolysis & Thermolysin, pepsin & Oxygen radical absorbance capacity & Huang et al. (2010) \\
\hline Enzymatic hydrolysis & $\begin{array}{l}\text { Protamex, alcalase, trypsin, } \\
\text { pepsin, neutrase, flavorzyme }\end{array}$ & ACE-inhibitory & Lee et al. (2010) \\
\hline Acid hydrolysis & $0.03 \mathrm{~N} \mathrm{HCl}$ & ACE-inhibitory & Lee et al. (2010) \\
\hline Enzymatic hydrolysis & thermolysin, pepsin & Antioxidant & Shen et al. (2010) \\
\hline Enzymatic hydrolysis & Thermolysin & ACE-inhibitory & Lei et al. (2011) \\
\hline Acid hydrolysis & $0.03 \mathrm{~N} \mathrm{HCl}$ & Antioxidant & Kim et al. (2012) \\
\hline Enzymatic hydrolysis & $\begin{array}{l}\text { protamex, alkalase, trypsin, } \\
\text { neutrase, flavorzyme, } \\
\text { maxazyme, collupulin, } \\
\text { protex, promod } 278, \alpha- \\
\text { chymotrypsin }\end{array}$ & Antioxidant & Kim et al. (2012) \\
\hline Acid hydrolysis & $0.03 \mathrm{~N} \mathrm{HCl}$ & Cytotoxic & Moon et al. (2013) \\
\hline Enzymatic hydrolysis & $\begin{array}{l}\text { Neutrase, alcalase, protamex, } \\
\text { protex } 6 \mathrm{~L}, \text { flavorzyme, } \alpha- \\
\text { chymotrypsin, trypsin, } \\
\text { collupulin }\end{array}$ & Cytotoxic & Moon et al. (2013) \\
\hline Enzymatic hydrolysis & Trypsin, ficin & Antimicrobial & Zohreh et al. (2014) \\
\hline Auto-cleave hydrolysis & $\begin{array}{l}0.2 \text { mmol }-1 \text { tris }(2- \\
\text { carboxyethyl) phosphine in } \\
\text { citrate-phosphate buffer }\end{array}$ & Antioxidant, Mineral chelating & Moon et al. (2014) \\
\hline Enzymatic hydrolysis & Promod 278P & Cytotoxic, Antioxidant & Moon et al. (2017) \\
\hline Enzyme hydrolysis & $\begin{array}{l}\text { Promod 278P, thermolysin, a } \\
\text { combination of promod } \\
\text { 278P-thermolysin }\end{array}$ & Antioxidant, Anticancer & Lee et al. (2017) \\
\hline Enzyme hydrolysis & Pepsin & Immunomodulatory & Liu et al. (2017) \\
\hline Enzymatic hydrolysis & Pepsin, trypsin & Anti-inflammatory & Wang et al. (2017) \\
\hline In Silico hydrolysis & $\begin{array}{l}19 \text { enzymes and } 1 \text { enzyme } \\
\text { combination }\end{array}$ & Potent ACE-inhibitory & Yu et al. (2019) \\
\hline Enzymatic hydrolysis & Pepsin & Antimicrobial & Ma et al. (2020) \\
\hline
\end{tabular}

functional foods, aiming to enhance the antioxidant properties of foods to prevent oxidation reactions during food processing, which would benefit human nutrition and health. Lee et al. (2017) reported that ovotransferrin hydrolysates obtained from promod 278P (OTH-P), thermolysin (OTH-T), and the combination of promod 278Pthermolysin (OTH-PT) had strong antioxidant properties. The DPPH radical scavenging activities of the hydrolysates were OTH-P 35.04\%, OTH-T 41.49\%, OTH-PT 43.53\%, and the ABTS-scavenging activities were OTH-P $47.23 \%$, OTO-T $62.36 \%$, OTH-PT $60.60 \%$ at $2 \mathrm{mg} / \mathrm{mL}$ concentration.

\section{ACE-inhibitory activity}

The ACE-inhibitory activity of the native ovotransferrin has not been reported yet. However, peptides derived from ovotransferrin were reported to have strong ACE-inhibitory activity. Moon et al. (2017) investigated the ACE-inhibitory activity of promod 278P hydrolysates of ovotransferrin and reported that the hydrolyzed products exhibited a strong ACE-inhibitory activity. Lee et al. (2010) reported that ovotransferrin hydrolysates produced with trypsin, acid, and pepsin reduced the activity of ACE by $60.2 \%, 55.8 \%$, and $42.6 \%$, respectively. Also, the ACEinhibitory peptide (Lys-Val-Arg-Glu-Gly-Thr-Thr-Tyr) of ovotransferrin significantly reduced the blood pressure in spontaneously hypertensive mice (Lee et al., 2006). They suggested that Lys-Val-Arg-Glu-Gly-Thr-Thr-Tyr acted as 
a pro-drug of Lys-Val-Arg-Glu-Gly-Thr to inhibit ACE activity in vivo. Majumder et al. (2015) reported that the ovotransferrin-derived peptide Ile-Arg-Trp increased the ACE-inhibitory activity but decreased the expression of proinflammatory gene in mesenteric arteries of spontaneously hypertensive rats. Recently, Yu et al. (2019) reported that Glu-Trp-Leu obtained from in silico hydrolysis of ovotransferrin using 19 enzymes and one enzyme combination showed potent ACE-inhibitory activity. Moon et al. (2017) reported that the promod 278P hydrolysate of ovotransferrin had $76.82 \pm 1.28 \%$ ACE-inhibitory activity at $10 \mathrm{mg} / \mathrm{mL}$ concentration.

\section{Cytotoxic activity}

The hydrolysates of ovotransferrin showed a cytotoxic effect on various cancer cells. Ovotransferrin hydrolyzed with promod $278 \mathrm{P}$ expressed over $90 \%$ of cytotoxicity against human breast adenocarcinoma (MCF-7), human colon adenocarcinoma (HT-29), human colon adenocarcinoma (LoVo), human cervix adenocarcinoma (HeLa), and human liver hepatoblastoma (HepG2) cancer cell lines (Moon et al., 2017). Majumder et al. (2013) reported that peptides (Ile-Arg-Trp and Ile-Gln-Trp) derived from ovotransferrin attenuated tumor necrosis factor, inflammatory responses, and oxidative stress in endothelial cells. Moon et al. (2013) reported that the hydrolysates of ovotransferrin with trypsin and neutrase showed over 90\% cytotoxicity against cervical cancer cell lines and those with trypsin, protamex, collupulin MG and protex 6L caused 95, 96,87 , and $86 \%$ growth inhibition, respectively, in stomach cancer cell lines. The hydrolysis of ovotransferrin by promod 278P (OTH-P), thermolysin (OTH-T), and its combination (OTH-PT) increased the cytotoxic activity against various cancer cells and the hydrolysate of OTHPT exhibited the strongest cytotoxic activities against human stomach adenocarcinoma (AGS), LoVo, HT-29 and HeLa with IC50 values $0.79,0.78,0.92$ and $0.78 \mathrm{mg} / \mathrm{mL}$, respectively (Lee et al., 2017).

\section{Immunomodulatory and anti-inflammatory activity}

The peptides derived from ovotransferrin also exhibited immunomodulatory activities. The Ile-Arg-Trp peptide of ovotransferrin exhibited a modulatory effect on vascular smooth muscle cells against Angiotensin II stimulation, superoxide production, and inflammation (Liao et al., 2016). Liu et al. (2017) reported that pepsin hydrolysates of ovotransferrin inhibited the maturation of lipopolysaccharide-induced bone marrow-derived dendritic cells (BMDCs) generated from $\mathrm{KM}$ mice by reducing the expression of MHC-II, CD83, and CD86, and the production of TNF- $\alpha$, lipopolysaccharide (IL-12p70), and
RANTES. Also, the pepsin hydrolysates of ovotransferrin impaired the ability of lipopolysaccharide-stimulated $\mathrm{BMDCs}$ to induce allogeneic $\mathrm{T}$ lymphocyte proliferation and decreased the production of IFN- $\gamma$ by activating $\mathrm{T}$ cells. Yi et al. (2017) reported that Ile-Arg-Trp inhibited the $\mathrm{H}_{2} \mathrm{O}_{2}$-induced cytotoxicity and production of reactive oxygen spices (ROS). Also, Ile-Arg-Trp normalized mitochondrial membrane potential and inhibited the activation of caspase-9 and caspase-3, and exhibited a protective effect against oxidative stress. Ma et al. (2019) showed that Ile-Arg-Trp and Ile-Gln-Trp derived from ovotransferrin improved intestinal health by regulating microbial composition in the colon, colonic inflammation caused by Citrobacter rodentium, and serum metabolism of mouse models. Wang et al. (2017) reported that ovotransferrinderived peptides also exerted anti-inflammatory effects. Dipeptides (Cys-Arg, Phe-Ile, His-Cys, Leu-Leu, and MetLys) of ovotransferrin decreased IL-8 secretion, inhibited gene expression of 5 pro-inflammatory cytokines (TNF- $\alpha$, IL-8, IL-6, IL-1ß, IL-12). In contrast, the gene expression of anti-inflammatory cytokines IL-10 in Caco-2 cells was promoted by those 5 di-peptides. Cys-Arg and His-Cys were also reported to inhibit the phosphorylation of p-JNK and p-p-38 (Wang et al., 2017). In an in vivo study, the IleArg-Trp from ovotransferrin inhibited lipopolysaccharide (LPS)-induced peritonitis in rat models by inhibiting the synthesis of serum TNF and IL-8 and myeloperoxidase. Also, Ile-Arg-Trp treatment increased the Shannon index and decreased the Simpson index and suggested that modification of gut microbial diversity due to the potential ability of Ile-Arg-Trp to enhance the barrier properties of the intestine (Jiao et al., 2019) (Table 2).

\section{Applications of ovotransferrin and its derived peptides}

The presence of a wide spectrum of functional properties in the ovotransferrin and its derived peptides suggests their potential applications in food, as well as medical and pharmaceutical industries, are very high.

\section{Food and nutraceutical applications}

As mentioned earlier, due to antimicrobial and antioxidant activities, ovotransferrin and its derived peptides can be used as functional food ingredients to enhance human health. However, few practical applications have been reported. Seol et al. (2009) developed a $\kappa$-carrageenanbased film containing ovotransferrin combined with EDTA and used it as a packaging material to extend the shelf-life of the fresh chicken breast by inhibiting E. coli. You et al. (2014) reported that ovotransferrin-catechin conjugate 
Table 2 Ovotransferrin-derived peptides and their activities

\begin{tabular}{lll}
\hline Ovotransferrin peptide & Activity & References \\
\hline OTAP-92 (Leu109-Asp200) & Antimicrobial & Ibrahim et al. (2000) \\
Asp-Gln-Lys-Asp-Glu-Tyr-Glu-Leu-Leu & Antiviral & Giansanti et al. (2005) \\
Lys-Asp-Leu-Leu-Phe-Lys & & \\
Lys-Val-Arg-Glu-Gly-Thr-Thr-Tyr & ACE-inhibitory & Lee et al. (2006) \\
Trp-Asn-Ile-Pro & Antioxidant & Shen et al. (2010) \\
Gly-Trp-Asn-Ile & & \\
Ile-Arg-Trp & Oxygen radical absorbance capacity & Huang et al. (2010) \\
Ile-Arg-Trp & Anticancer & Majumder et al. (2013) \\
Ile-Gln-Trp & & Majumder et al. (2015) \\
Ile-Arg-Trp & ACE-inhibitory & Jiao et al. (2019), Liao et al. (2016), Ma et al. (2019) \\
Ile-Arg-Trp & Modulatory, ACE-inhibitory & Ma et al. (2019) \\
Ile-Gln-Trp & Immunomodulatory & Wang et al. (2017) \\
Cys-Arg & Anti-inflammatory & \\
Phe-Ile & & \\
His-Cys & & \\
Leu-Leu & & \\
Met-Lys & & \\
\hline
\end{tabular}

could be used as an antioxidant in functional foods. Recently, ovotransferrin was used to fabricate gum arabic particles by electrostatic interactions, hydrophobic interactions, and hydrogen bonding to employ as a surfactantfree food-grade Pickering emulsion with applications of protein-polysaccharide nanocomplexes (Wei and Huang, 2019a). Ovotransferrin-glucose conjugate (OGC) and ovotransferrin-lactose conjugate (OLC) obtained from Maillard reaction through glucosylation and galactosylation respectively, were tested for their functional properties. However, the covalently bound saccharides weakened the emulsifying ability of ovotransferrin fibrils and had larger emulsion droplet sizes of OGC fibrils-stabilized emulsions than OLC fibrils-stabilized emulsions and OGC exerted the lowest viscosity and storage modulus. Hence, the rheological properties of oil emulsions stabilized by ovotransferrin fibrils were dependent on the nature of the covalently bound saccharides (Wei and Huang, 2019b).

\section{Medical and pharmaceutical applications}

As described previously, ovotransferrin and its derived peptides have great potential to be applied in medical and pharmaceutical industries as antimicrobial, antiviral, anticancer, and antihypertensive agents. Ovotransferrin and the peptides from ovotransferrin, Ile-Arg-Trp, Ile-Gln-Trp, and Lys-Val-Arg-Glu-Gly-Thr, showed ACE-inhibitory, antihypertensive, anti-inflammatory, and antioxidant properties, and thus could be used as a clinical treatment for cardiovascular diseases (Chen et al., 2017). Also, ovotransferrin was suggested to be used as an antibiotic substitute to prevent chlamydiosis (Van Droogenbroeck et al., 2008). Van Droogenbroeck et al. (2011) clinically used ovotransferrin as an antimicrobial agent to treat turkey and successfully reduced the amount of Chlamydia psittaci, Ornithobacterium rhinotracheale, and avian metapneumovirus. Abdallah and Chahine (1999) suggested using ovotransferrin as a metal supplementing agent for humans due to its iron-binding and transportation capacity. Moreover, ovotransferrin has been reported to have an osteogenic activity that promotes bone formation while preventing bone resorption. Ovotransferrin can also act as a therapeutic and anabolic agent to prevent osteoporosis and promote bone repair (Shang and $\mathrm{Wu}, 2018$ ). Son et al. (2018) investigated angiotensin II-stimulated insulin of triACE-inhibitory peptides of ovotransferrin (Ile-Arg-Trp, Ile-Gln-Trp, Leu-Lys-Pro) and reported that only Ile-ArgTrp decreased insulin-stimulated glucose uptake in ratderived skeletal muscle cells. Also, egg white ovotransferrin suppressed the Receptor Activator of Nuclear Factor $\kappa \mathrm{B}$ ligand (RANKL) and inhibited osteoclast differentiation, which promoted bone health (Shang and Wu, 2019). Wei et al. (2019) reported that the formation of a hetero protein complex between ovotransferrin and lysozyme stabilized Pickering emulsions, which was an effective delivery tool for hydrophobic nutraceuticals. Ovotransferrin is also tested as a therapeutic agent to treat retained placenta, reproductive tract infection, and inflammation such as metritis to improve the health of the reproductive tract and fertility of cows (Talukder et al., 2019). 


\section{Egg allergenicity and safety aspects}

Eggs are considered as one of the main allergenic foods that account for $38.3 \%$ of all food allergies (Sakai et al., 2015 ) and its estimated prevalence is $0.2-7.0 \%$ (Rona et al., 2007). As described by Ebbehoj et al. (1995) egg white proteins, including ovomucoid, ovalbumin, ovotransferrin, and lysozyme, are the major allergenic proteins in egg white. As eggs are consumed as a functional food, the avoidance of allergenicity is very important. Scientific evidence revealed that various processing procedures and safety measures can be applied to reduce/eliminate allergenicity of eggs. Hoffman (1983) reported that allergenicity can be simply decreased by boiling eggs. Also, the combination of heat and enzyme treatments reduced the allergenicity of pasteurized eggs without significantly changing their texture and flavor. Furthermore, the enzymatic hydrolysis of proteins into peptides and amino acid monomers is an effective method to eliminate the egg allergenicity. Also, the allergenicity of egg proteins can be decreased by processing egg with other food ingredients: heating of eggs with wheat flour made ovomucoid insoluble by forming intermolecular disulfide protein-polysaccharide polymers and became less extractable and minimized allergenicity (Kato et al., 2001).

\section{Summary and future research approaches}

This study reviewed the structure, separation, and functional properties of ovotransferrin and the production of functional peptides from ovotransferrin. Ovotransferrin and its derived peptides have been recognized for their excellent functional properties. Among the functional properties, antimicrobial, antioxidant, and ACE-inhibitory activities were highly recognized and investigated. Although the in vitro experiments for the functional properties of ovotransferrin and its derived peptides were established well, further researches are needed to investigate the bioactivities of ovotransferrin products to apply in biosystems via food, animal, and clinical trials. The antimicrobial activity of ovotransferrin and its derived peptides can be used as a natural preservative to prevent microbial spoilage in foods while enhancing nutritional value. Also, due to the presence of antioxidant properties, ovotransferrin and its bioactive peptides have future potential applications in food processing to prevent undesirable oxidative reactions caused by substances such as metal ions. Also, ovotransferrin and its functional hydrolysates can be used as a pharmaceutical agent that can regulate high blood pressure through its ACE-inhibitory activity. The industrial purification and application of ovotransferrin and its peptides are not implemented yet, but simple, economical, and scalable separation methods for ovotransferrin are already available, but the identification of particular bioactive peptides of ovotransferrin with their sequences and their application needs further studies. Because various processing methods, such as heating, can influence the physiological and functional properties of the protein, further research is needed to analyze the activities of ovotransferrin and its derivatives in vivo. The determination of novel functional properties creates new research approaches for the ovotransferrin and its derived peptides even though most of the approaches used single enzyme hydrolysis of ovotransferrin and few pieces of research were carried out with enzyme combinations. The separation of ovotransferrin and the identification of its derived peptides as a food additive creates a novel trend in the food industry and commercial production opportunities with economical value as well. Although it is well known that ovotransferrin is an iron carrier protein in the animal body, little work has been done to use it as an iron supplementing agent for humans, which needs further attention. The ovotransferrin nanofibrils and their activities were identified as novel applications of ovotransferrin with advantages in the food and pharmaceutical industries, this area needs further studies.

Acknowledgements No funds were received for the completion of this review manuscript.

\section{References}

Abdallah FB, Chahine JM. Transferrins, the mechanism of iron release by ovotransferrin. European Journal of Biochemistry. 263: 912-920 (1999).

Abeyrathne EDNS, Lee HY, Ahn DU. Egg white proteins and their potential use in food processing or as nutraceutical and pharmaceutical agents - A review. Poultry Science. 92: 3292-3299 (2013a).

Abeyrathne EDNS, Lee HY, Ahn DU. Separation of ovotransferrin and ovomucoid from the chicken egg white. Poultry Science. 93: 1010-1017 (2014b).

Abeyrathne EDNS, Lee HY, Ahn DU. Sequential separation of lysozyme, ovomucin, ovotransferrin, and ovalbumin from the egg white. Poultry Science. 93: 1001-1009 (2014a).

Abeyrathne EDNS, Lee HY, Ham JS, Ahn DU. Separation of ovotransferrin from chicken egg white without using solvents. Poultry Science. 92: 1091-1097 (2013b).

Acero-Lopez A, Ullah A, Offengenden M, Jung S, Wu J. Effect of high-pressure treatment on ovotransferrin. Food Chemistry. 135: 2245-2252. (2012).

Al-Mashikhi SA, Nakai S. Separation of ovotransferrin from egg white by immobilized metal-affinity chromatography. Agricultural and Biological Chemistry. 51: 2881-2887 (1987).

Awade AC, Moreau S. Mollé D, Brule D, Maubois JL. Two-step chromatographic procedure for the purification of hen egg-white ovomucin, lysozyme, ovotransferrin, and ovalbumin and characterization of purified proteins. Journal of Chromatography A. 677: 279-288 (1994). 
Azari P, Baugh R. A simple and rapid procedure for the preparation of large quantities of pure ovotransferrin. Archives of Biochemistry. 118: 138-144 (1967).

Baron F, Jan S, Gonnet F, Pasco M, Jardin J, Giudici B, Gautier M, Guérin-Dubiard C, Nau F. Ovotransferrin plays a major role in the strong bactericidal effect of egg white against the Bacillus cereus group. Journal of Food Protection. 77: 955-962 (2014).

Chang C, Lahti T, Tanaka T, Nickerson MT. Egg proteins: fractionation, bioactive peptides, and allergenicity. Journal of the Science of Food and Agriculture. 98: 5547-5558. (2018).

Charter E, Lagarde G. Natural antimicrobial systems/lysozyme and other proteins in eggs. 1. pp 1582-1587. In: Encyclopedia of Food microbiology. Robinson R. Academic Press Elsevier. San Diego. (2004).

Chen S, Jiang H, Peng H, Wu X, Fang J. The utility of ovotransferrin and ovotransferrin-derived peptides as possible candidates in the clinical treatment of cardiovascular diseases. Oxidative Medicine and Cellular Longevity. Article ID 6504518 (2017).

Chiurciu C, Chiurciu V, Oporanu M, Pătrascu IV, Mihai I, Tablică M, Cristina RT. PC2 Ovotransferrin: Characterization and alternative immunotherapeutic activity. Evidence-Based Complementary and Alternative Medicine. Article ID 8671271 (2017).

Chung MCM, Chan SL, Shimizu S. Purification of transferrin and lactoferrin using DEAE Affi-gel blue. International Journal of Biochemistry. 23: 609-616 (1991).

Cooper CA, Tizard ML, Stanborough T, Moore SC, Chandry PS, Jenkins KA, Wise TG, O’Neil TE, Layton DS, Morris KR Moore RJ, Fegan N, Doran TJ. Overexpressing ovotransferrin and avian B-defensin-3 improves the antimicrobial capacity of chickens and poultry products. Transgenic Research. 28: 51-76 (2019).

Croguennec T, Nau F, Pezennec S, Piot M, Brule G. Two-step chromatographic procedure for the preparation of hen egg-white ovotransferrin. European Food Research and Technology. 2012: 296-301 (2001).

Desert C, Guerin-Dubiard C, Nau F, Jan G, Val F, Mallard J. Comparison of different electrophoretic separations of hen egg white proteins. Journal of Agricultural and Food Chemistry. 49: 4553-4561 (2001).

Ebbehoj K, Dahl AM, Frokier H, Norgaad A, Poulsen LK, Barkholt V. Purification of egg-white allergens. Allergy. 50: 133-141 (1995).

Fraenkel-Conrat H, Feeney R. The metal-binding activity of conalbumin. Archives of Biochemistry and Biophysics. 29: 101-113 (1950).

Giansanti F, Leboffe L, Angelucci F, Antonini G. The nutraceutical properties of ovotransferrin and its potential utilization as a functional food. Nutrients. 7: 9105-9115 (2015).

Giansanti F, Massucci MT, Giardi MF, Nozza F, Pulsinelli E, Nicolini C, Botti D, Antonini G. Antiviral activity of ovotransferrin derived peptides. Biochemical and Biophysical Research Communication. 331: 69-73 (2005).

Giansanti F, Rossi P, Massucci MT, Botti D, Antonini G, Valenti P, Seganti L. Antiviral activity of ovotransferrin discloses an evolutionary strategy for the defensive activities of lactoferrin. Biochemistry and Cell Biology. 80: 125-130 (2002).

Hoffman DR. Immunochemical identification of the allergens in egg white. Journal of Allergy Clinical Immunology. 71: 481-486. (1983).

Huang WY, Majumder K, Wu J. Oxygen radical absorbance capacity of peptides from egg white protein ovotransferrin and their interaction with phytochemicals. Food Chemistry. 123: 635-641 (2010).

Ibrahim HR, Imranul HM, Aoki T. Ovotransferrin possesses SODlike superoxide anion scavenging activity that is promoted by copper and manganese binding. International Journal of Biological Macromolecules. 41: 631-640 (2007).
Ibrahim HR, Iwamori E, Sugimoto Y, Aoki T. Identification of a distinct antibacterial domain within the N-lobe of ovotransferrin. Biochimica et Biophysica Acta. 1401: 289-303 (1998).

Ibrahim HR, Kiyono T. Novel anticancer activity of the autocleaved ovotransferrin against human colon and breast cancer cells. Journal of Agriculture and Food Chemistry. 57: 11383-11390 (2009).

Ibrahim HR, Sugimoto Y, Aoki T. Ovotransferrin antimicrobial peptide (OTAP-92) kills bacteria through a membrane damage mechanism. Biochimica et Biophysica Acta. 1523: 196-205 (2000).

Ibrahim HR. Ovotransferrin. pp 211-226. In: Natural Food Antimicrobial Systems. Naidu AS. FL: CRC Press. (2000).

Ji S, Ahn DU, Zhao Y, Li K, Sheng L, Huang X. An easy and rapid separation method for five major proteins from egg white: successive extraction and MADI-TOF-MS identification. Food Chemistry. 315: (2020) 126207.

Jiao H, Zhang Q, Lin Y, Gao Y, Zhang P. The ovotransferrin-derived peptide IRW attenuates lipopolysaccharide-induced inflammatory responses. BioMed Research International. Article ID 8676410 (2019).

Kato Y, Oozawa E, Matsuda T. Decrease in antigenic and allergenic potentials of ovomucoid by heating in the presence of wheat flour: dependence on wheat variety and intermolecular disulfide bridges. Journal of Agricultural and Food Chemistry. 49: 3661-3665 (2001).

Keung WM, Azari P, Phillips JL. Structure and function of ovotransferrin. The Journal of Biochemical Chemistry. 257: 1177-1183 (1982).

Kim J, Moon SH, Ahn DU, Paik HD, Park E. Antioxidant effects of ovotransferrin and its hydrolysates. Poultry Science. 91: 2747-2754 (2012).

Ko KY, Ahn DU. An economic and simple purification procedure for the large-scale production of ovotransferrin from the egg white. Poultry Science. 87: 1441-1450 (2008).

Ko KY, Mendonca AF, Ahn DU. Effect of ethylenediaminetetraacetate and lysozyme on the antimicrobial activity of ovotransferrin against Listeria monocytogenes. Poultry Science. 87: 1649-1658 (2008a).

Ko KY, Mendonca AF, Ahn DU. Influence of zinc, sodium bicarbonate, and citric acid on the Antibacterial Activity of ovotransferrin against Escherichia coli $0157: \mathrm{H} 7$ and Listeria monocytogenes in model systems and ham. Poultry Science. 87: 2660-2670 (2008b).

Kovacs-Nolan JKN, Phillips M, Mine Y. Advances in the value of eggs and egg components for human health. Journal of Agriculture and Food Chemistry. 53: 8421-8431 (2005).

Kurokawa H. Mikami B, Hirose M. Crystal structure of di-ferric hen ovotransferrin at $2.4 \AA$ resolution. Journal of Molecular Biology. 254: 196-207 (1995).

Lee JH, Ahn DU, Paik HD. In Vitro immune-enhancing activity of ovotransferrin from egg white via MAPK signaling pathways in RAW 264.7 macrophages. Korean Journal for Food Science of Animal Resources 38: 1226-1236 (2018).

Lee JH, Moon SH, Kim HS, Park E, Ahn DU, Paik HD. Antioxidant and anticancer effects of functional peptides from ovotransferrin hydrolysates. Journal of the Science of Food and Agriculture. 97: 4857-4864 (2017).

Lee NK, Ahn DU, Park KK, Paik HD. Ovotransferrin angiotensinconverting enzyme (ACE) and optimization for the production of ovotransferrin hydrolysates. Korean Journal for Food Science of Animal Resources. 30: 286-290 (2010).

Lee NY, Cheng JT, Enomoto T, Nakano Y. One peptide derived from hen ovotransferrin as pro-drug to inhibit the angiotensinconverting enzyme. Journal of Food and Drug Analysis. 14: 31-35 (2006). 
Lei B, Majumder $\mathrm{K}$, Shen $\mathrm{S}$, Wu J. Effect of sonication on thermolysin hydrolysis of ovotransferrin. Food Chemistry. 124: 808-815 (2011).

Liao W, Chakrabarti S, Davidge ST, Wu J. Modulatory effects of egg white ovotransferrin-derived tripeptide IRW (Ile-Arg-Trp) on vascular smooth muscle cells against angiotensin II stimulation. Journal of Agricultural and Food Chemistry. 64: 7342-7347 (2016).

Lin LN, Mason AB, Woodworth RC, Brandts JF. Colorimetric studies of serum transferrin and ovotransferrin. Estimates of domain interactions, and study of the kinetic complexities of ferric ion binding. Biochemistry. 33: 1881-1888 (1994).

Liu L, Xu M, Tu Y, Du H, Zhou Y, Zhu G. Immunomodulatory effect of protease hydrolysates from ovotransferrin. Food and Function. 8: 1452-1459 (2017).

Ma B, Guo Y, Fu X, Jin Y. Identification and antimicrobial mechanisms of a novel peptide derived from egg white ovotransferrin hydrolysates. LWT - Food Science and Technology. 131: 1-9 (2020).

Ma Y, Ding S, Liu G, Fang J, Yan W, Duraipandiyan V, Al-Dhabi NA, Esmail GA, Jiang $\mathrm{H}$. Egg protein transferrin-derived peptides IRW and IQW regulate Citrabacter rodentium-induced, inflammation-related microbial and metabolomic profiles. Frontiers in Microbiology.10: 643 (2019).

Majumder K, Chakrabarthi S, Davidge ST, Wu J. Structure and activity study of egg protein ovotransferrin derived peptides (IRW and IQW) on endothelial inflammatory response and oxidative stress. Journal of Agriculture and Food Chemistry. 61: 2120-2129 (2013)

Majumder K, Liang G, Chen Y, Guan L, Davidge ST, Wu J. Egg ovotransferrin-derived ACE-inhibitory peptide IRW increases ACE2 but decreases proinflammatory genes expression in the mesenteric artery of spontaneously hypertensive rats. Molecular Nutrition and Food Research. 59: 1735-1744 (2015).

Möller NP, Elisabeth K, Ahrens S, Roos N, Schrezenmeir J. Bioactive peptides and proteins from foods: indication for health effects. European Journal of Nutrition. 47: 171-182 (2008).

Moon SE, Lee JH, Lee YU, Paik JY, Ahn DU, Paik HD. Antioxidant, antimicrobial, and cytotoxic activities of ovotransferrin from the egg white. Korean Journal of Food Science and Animal Resources. 32: 612-617 (2012).

Moon SH, Lee JH, Ahn DU, Paik HD. In vitro antioxidant and mineral-chelating properties of natural and autocleaved ovotransferrin. Journal of the Science of Food and Agriculture. 95: 2065-2070 (2014)

Moon SH, Lee JH, Kim JH, Paik HD, Ahn DU. In vitro cytotoxic and ACE-inhibitory activities of promod 278P hydrolysate of ovotransferrin from the chicken egg white. Poultry Science. 96: 1982-1987 (2017).

Moon SH, Lee JH, Lee YJ, Chang KH, Paik JY, Ahn DU, Paik HD. Screening for cytotoxic activity of ovotransferrin and its enzyme hydrolysates. Poultry Science. 92: 424- 434 (2013).

Omana DA, Wang J, Wu J. Co-extraction of egg white proteins using ion-exchange chromatography from ovomucin removed egg whites. Journal of Chromatography B. 878: 1771-1776 (2010).

Rhodes MB, Azari PR, Feeney RE. Analysis, fractionation, and purification of egg white proteins with cellulose-cation exchanger. The Journal of Biochemical Chemistry. 230: 399-408 (1958).

Rona RJ, Keli T, Summers C, Gislason D, Zuidmeer L, Sodergren E, Sigurdardottir ST, Lindner T, Goldhahn K, Dahlstrom J, McBride D, Madsen C. The prevalence of food allergy: a meta-analysis. Journal of Allergy Clinical Immunology. 120: 638-646 (2007).

Sakai S, Adachi R, Teshima R. Detection and control of eggs as a food allergen. pp 313-340. In: Handbook of food allergen detection and control. Flanagan S. Woodhead Publishing. (2015).

Schade AL, Caroline L. 1944. Raw hen egg white and the role of iron in growth inhibition of Shigella dysenteriae, Staphylococcus aureus, Escherichia coli, and Saccharomyces cerevisiae. Science. 100: 14-15 (1944).

Seol KH, Lim DG, Jang A, Jo C, Lee M. Antimicrobial effect of $\kappa$ carrageenan-based edible film containing ovotransferrin in fresh chicken breast stored at $5^{\circ} \mathrm{C}$. Meat Science. 83: 479-483 (2009).

Shang N, Bhullar KS, Wu J. Ovotransferrin exhibits osteogenic activity partially via low-density lipoprotein receptor-related protein 1 (LRP 1) activation in MC3T3-E1 cells. Journal of Agricultural and Food Chemistry. 68: 9427-9435 (2020).

Shang N, Wu J. Egg white ovotransferrin attenuates RANKL-induced osteoclastogenesis and bone resorption. Nutrients 11(9): 1-13 (2019).

Shang N, Wu J. Egg white ovotransferrin shows osteogenic activity in osteoblast cells. Journal of Agricultural and Food Chemistry. 66: 2775-2782 (2018).

Shang N. The potential of egg white ovotransferrin as a functional food ingredient against osteoporosis. PhD thesis. University of Alberta, Edmonton, Alberta, Canada. (2019).

Shen S, Chahal B, Majumder K, You SJ, Wu J. Identification of novel antioxidative peptides derived from a thermolytic hydrolysate of ovotransferrin by LC-MS/MS. Journal of Agricultural and Food Chemistry. 58: 7664-767 (2010).

Shibusawa Y, Kihira S, Ito Y. One-step purification proteins from egg white using counter-current chromatography. Journal of Chromatography B. Biomedical Sciences and Applications. 709: 301-305 (1998)

Shibusawa Y, Lino S, Shindo H, Ito Y. Separation of chicken egg white proteins by high-speed countercurrent chromatography. Journal of Liquid Chromatography and Related Technologies. 24: 2007-2016 (2001).

Shimazaki Y, Takahashi A. Antibacterial activity of lysozymebinding proteins from the chicken egg white. Journal of Microbiological Methods. 154: 19-24 (2018).

Talukder J, Ray A, DuBourdieu DJ, Srivastava A, Lall R. Ovotransferrin treatment for the reproductive tract. U. S. Patent US 20190314460A1 (2019).

Tankrathok A, Daduang S, Patramanon R, Araki T, Thammasirirak S. Purification process for the preparation and characterizations of hen egg-white ovalbumin, lysozyme, ovotransferrin, and ovomucoid. Preparative Biochemistry and Biotechnology. 39: 380-399 (2009).

Vachier MC, Piot M, Awade AC. Isolation of hen egg-white lysozyme, ovotransferrin, and ovalbumin, using quaternary ammonium bound to a highly crosslinked agarose matrix. Journal of Chromatography B: Biomedical Applications. 664: 201-210 (1995).

Valenti P, Antonini G, Rossi Fanelli MR, Orsi N, Antonini E. Antibacterial activity of matrix-bound ovotransferrin. Antimicrobial Agents and Chemotherapy. 21: 840-841 (1982).

Valenti P, Antonini GRH, Visca P, Orsi N, Antonini E. Studies of the antimicrobial activity of ovotransferrin. International Journal of Tissue Reactions. 5: 97-105 (1983).

Valenti P, Visca P, Antonini G, Orsi N, Antonini E. The effect of saturation with $\mathrm{Zn}^{2+}$ and other metal ions on the antibacterial activity of ovotransferrin. Medical Microbiology and Immunology. 176: 123-130 (1987).

Valenti P, Visca P, Antonini G, Orsi N. Antifungal activity of ovotransferrin towards genus Candida. Mycopathologia. 89: 169-175 (1985)

Valenti P, Visca P, Antonini G, Orsi N. Interaction between lactoferrin and ovotransferrin and Candida cells. FEMS Microbiology Letters. 33: 271-275 (1986). 
Van Droogenbroeck C, Beeckman DSA, Harkinezhad T, Cox E, Vanrompay D. Evaluation of the prophylactic use of ovotransferrin against chlamydiosis in SPF turkeys. Veterinary Microbiology. 132: 372-378 (2008).

Van Droogenbroeck C, Dossche L, Wauman T, Van Lent S, Phan TTT, Beeckman DSA, Vanrompay D. Use of ovotransferrin as an antimicrobial in turkey naturally infected with Chlamydia psittaci, avian metapneumovirus, and Ornithobacterium rhinotracheale. Veterinary Microbiology. 153: 257-263 (2011).

Wang X, Zhao Y, Yao Y, Xu M, Du H, Zhang M, Tu Y. Antiinflammatory activity of di-peptides derived from ovotransferrin by simulated peptide-cut in TNF- $\alpha$-induced Caco- 2 cells. Journal of Functional Foods. 37: 424-432 (2017).

Warner RC, Weber I. The preparation of crystalline conalbumin. The Journal of Biological Chemistry. 191: 173-180 (1951).

Wei Z, Cheng Y. Huang Q. Heteroprotein complex formation of ovotransferrin and lysozyme: fabrication of food-grade particles to stabilize Pickering emulsions. Food Hydrocolloids. 96: 190-200 (2019).

Wei Z, Huang Q. Edible Pickering emulsions stabilized by ovotransferrin-gum Arabic particles. Food Hydrocolloids. 89: 590-601 (2019a).

Wei Z, Huang Q. Modification of ovotransferrin by Maillard reaction: consequences for structure, fibrillation, and emulsifying property of fibrils. Food Hydrocolloids. 97: (2019b) 105186.

Wenn RV, Williams J. The isoelectric fractionation of hen's-egg ovotransferrin. Biochemical Journal. 108: 69-74 (1968).

Williams J, Elleman TC, Kingston IB, Wilkins AG, Kuhn KA. The primary structure of hen ovotransferrin. European Journal of Biochemistry. 122: 297-303 (1982).

Williams J. A comparison of glycopeptides from the ovotransferrin and serum transferrin of the hen. Biochemical Journal. 108: 57-67 (1968).

Wu J, Acero-Lope A. Ovotransferrn: structure, bioactivities, and preparation. Food Research International. 46: 480-487 (2012).

Wu J, Eggs, and Egg Products Processing. pp. 437-455. In: Food Processing: Principles and Applications. Clark S, Jung S. Lamsal B. Wiley (2014).

Xie H, Huff GR, Huff WE, Balog JM, Rath NC. Effects of ovotransferrin on chicken macrophages and heterophil- granulocytes. Development and Comparative Immunology. 26: 805-815 (2002)

Yi J, Zhao J, Wu J. Egg ovotransferrin-derived IRW exerts a protective effect against $\mathrm{H}_{2} \mathrm{O}_{2}$-induced oxidative stress in Caco2 cells. Journal of Functional Foods. 39: 160-167 (2017).

You J, Luo Y, Wu J. Conjugation of ovotransferrin with catechin shows improved antioxidant activity. Journal of Agricultural and Food Chemistry. 62: 2581-2587 (2014).

You SJ, Udenigwe CC, Aluko RE, Wu J. Multifunctional peptides from egg white lysozyme. Food Research International. 43: 848-855 (2010).

Yu S, Chen Y, Zhao W, Zheng F, Ding L, Liu J. Novel ACE inhibitory tripeptides from ovotransferrin using bioinformatics and peptidomics approaches. Scientific Reports. 9: 17434 (2019).

Zhang Q, Tan W, Yang L, Lu M, Dong S, Liu X, Duan X. Multiomics analysis of the effects of egg ovotransferrin on the gut environment in mice: mucosal gene expression, microbiota composition, and intestinal structural homeostasis. Molecular Nutrition and Food Research. 64: 1901024 (2020).

Zhou Y, Tang Q, Du H, Tu Y, Wu S, Wang W, Xu M. Antiviral effect of ovotransferrin in mouse peritoneal macrophages by upregulating type I interferon expression. Food and Agricultural Immunology. 29: 600-614 (2018).

Zhu G, Jiang Y, Yao Y, Wu N, Luo J, Hu M, Tu Y, Xu M. Ovotransferrin ameliorates the dysbiosis of immunomodulatory function and intestinal microbiota induced by cyclophosphamide. Food and Function. 10: 1109-1122 (2019).

Zhu G, Luo J, Du H, Jiang Y, Tu Y, Yao Y, Xu M. Ovotransferrin enhances intestinal immune response in cyclophosphamideinduced immunosuppressed mice. International Journal of Biological Macromolecules. 120: 1-9 (2018).

Zohreh T, Masoud H, Mahmoud A. Antimicrobial properties of bioactive peptides derived from trypsin and ficin treated ovotransferrin. Molecular Biology Research Communications. 3: 298 (2014).

Publisher's Note Springer Nature remains neutral with regard to jurisdictional claims in published maps and institutional affiliations. 